\title{
Role of CXCR4 and MMP-9 Expressions with Regional Cervical Lymph Nodes Metastatic Status in Papillary Thyroid Carcinoma
}

\author{
Nurdhani Hi Djafar, Etty Hary Kusumastuti, Alphania Rahniayu \\ Department of Anatomical Pathology, Faculty of Medicine, Universitas Airlangga - Dr. Soetomo Hospital, Surabaya, Indonesia
}

\section{ARTICLE INFO}

Received : September 2020

Reviewed : December 2020

Accepted : March 2021

\section{Keywords:}

CXCR4, lymph node metastasis, MMP-9, papillary thyroid carcinoma

*Corresponding author: Etty Hary Kusumastuti Department of Anatomical Pathology, Faculty of Medicine, Universitas Airlangga - Dr. Soetomo Hospital, Surabaya, Indonesia Etty_pa@yahoo.com

\begin{abstract}
A BSTRACT
Background: Papillary thyroid carcinoma (PTC) that has metastasized has a higher risk because of the poor prognosis, ranging from decreased quality of life of the patient to death. There is a need for markers that can understand the image of the tumor so that it can predict earlier the level of aggressiveness and the ability of tumor cells to metastasize. Tumor cells that proliferate excessively will cause CXCR4 expression. Activation of CXCR4 will increase MMP-9 via the MAPK/ ERK line. MMP-9 will degrade the extracellular matrix causing tumor cell metastasis. This study is aimed to analyze the correlation of CXCR4 and MMP-9 expressions with lymph node metastasis (LNM) in PTC.
\end{abstract}

Methods: This cross-sectional analytic observational study was conducted in 43 PTC samples taken from the Anatomical Pathology Laboratory of Dr. Soetomo Hospital from January 2011 to December 2018. The samples were divided into 2 groups based on the regional cervical LNM status. Immunohistochemical staining was used to assess the expression of CXCR4 and MMP-9. Spearman test was used to analyze the correlation of CXCR4 and MMP expression with cervical LNM status.

Results: The expression of CXCR4 and MMP-9 was higher in the papillary thyroid carcinoma group with cervical regional LNM $(p=0.007$ and $p=0.030)$. There was a significant positive relationship between CXCR4 and MMP-9 expressions with regional LNM status with $r s=0.468$ and $p=0.001(p<0.05)$.

Conclusions: Expression of CXCR4 and MMP-9 was proven to correlate with regional cervical LNM status in papillary thyroid carcinoma. Both can be used as predictive markers for metastasis in papillary thyroid carcinoma.

\section{INTRODUCTION}

Thyroid cancer is the most common endocrine malignancy with increased incidence in the last 20-30 years. According to the data from Global Cancer Statistic (GLOBOCAN) 2018, thyroid carcinoma is ranked ninth as the highest incident globally with approximately 567.000 cases [1,2]. In 2013, Basic Health Research conducted by the Health Ministry of Indonesia showed that thyroid carcinoma was ranked fifth as the most common malignancy. In 2013, there were 147 new thyroid cancer cases in Indonesia [3].

Until now, it is estimated that $30-80 \%$ of papillary thyroid carcinoma (PTC) patients have regional lymph node metastasis (LNM), causing recurrence, distant metastasis, and decreased survival rate. Paulino et al. [4] stated that lymph node metastasis is associated with a bad prognosis and occurs in $>50 \%$ of PTC cases [4-6]. A previous study shows that LNM only affects recurrence, not survival. A large-scale case-control study shows that LNM and inadequate surgical excision are the two main factors associated with morbidity $[7,8]$.

Recently, studies showed that chemoattractant molecules secreted from target organs and specific patterns of chemoattractant receptors expressed on tumor cells can determine the goal of tumor metastasis. Therefore, the expression of these chemoattractant receptors can 
promote the migration of tumor cells in response to the gradient of chemoattractant molecules originating from the lymph nodes. The identification of these molecules and their receptors can be considered as markers for PTC which is likely to undergo LNM. The chemoattractant receptor candidate is a chemokine receptor. The chemokine that plays a role in tumor cell migration is CXCL12 which has a specific receptor, namely CXCR4 [9].

Binding between CXCL12 and CXCR4 will activate AKT and mitogen-activated protein kinase (MAPK)/ERK signaling which will increase the migration ability of tumor cells including PTC cells and induce angiogenesis [10-12]. The MAPK/ERK pathway then induces MMP-9 expression via NF-K $\beta$ activity [13]. Matrix metalloproteinases-9 (MMP-9) is a group of proteolytic enzymes which plays an important role in the progression of cancer cells, increasing tumor cell invasion and metastasis. It detaches tumor cells from their place origin and then invades the blood and lymphatic system through the surrounding stroma. All these processes are related to MMP-9 which has the function of degrading type IV collagen as the main component of the basement membrane $[12,14,15]$. This study was aimed to analyze the correlation of CXCR4 and MMP-9 in regional cervical lymph node metastasis in PTC.

\section{METHODS}

This study was an observational analytic study with a cross-sectional approach, conducted on PTC cases obtained from Anatomical Pathology Laboratory of Dr. Soetomo General Hospital archives from January 2011 to December 2018 which met the inclusion criteria. The inclusion criteria include the use of paraffin blocks from patients with classic variant PTC obtained from hemithyroidectomy or total thyroidectomy by including lymph node (LN) specimens or radical neck dissection (RND) regardless of the level of $L N$. The samples were divided into two groups consisting of 32 and 11 samples with and without regional cervical LNM, respectively.

Immunohistochemical staining was used to assess the expression of CXCR4 and MMP-9. Paraffin block was sliced for $4 \mu \mathrm{m}$ thin, deparaffinized, and rehydrated using 96\%, $80 \%$, and $70 \%$ of ethanol. It was then processed in a decloaking chamber using citrate buffer $(\mathrm{pH} \mathrm{6}$, temperature 950) for 45 minutes. Primary antibody CXCR4 (sc-53534, Santa Cruz Biotechnology, Inc.) diluted 1:200 and MMP9 (sc-13520, Santa Cruz Biotechnology, Inc.) diluted 1:200 were given overnight. A secondary antibody was then applied, and the samples were incubated for 20 minutes. Diaminobenzidine (DAB) was added, and counterstain was performed using Meyer Hematoxylin. CXCR4 and MMP-9 expressions were assessed using a percentage score. CXCR4 percentage score was divided into score 0 = stained in $0-10 \%$ tumor cells, score $1=$ stained in $10-50 \%$ tumor cells, and score 2 = stained in $>50 \%$ tumor cells. MMP-9 percentage score was divided into score 0 $=$ not stained or stained in $<5 \%$ tumor cells, score $1=$ stained in 5-25\% tumor cells, and score $3=$ stained in > $50 \%$ tumor cell. CXCR4 expression was observed in the cell membrane and cytoplasm of tumor cells, while MMP9 expression was observed in the cytoplasm of tumor cells. A binocular light microscope was used to observe the expression of CXCR4 and MMP-9.

The difference in the expression of CXCR4 and MMP9 in the two groups was carried out by the Mann Whitney test. The results of the Mann-Whitney test were said to be significantly different if the $p$-value was $<0.05$. Spearman correlation test was used to assess the association between regional cervical LNM status with CXCR4 and MMP-9 expressions. The results were considered significant if $p<0.05$.

\section{RESULTS}

In this study, the mean patient age was 47.7 years. The youngest patient was 19 years old while the oldest was 75 years old, and most patients were at the age of < 55 years (67.4). In the metastatic group, most were found in the age group of $<55$ years as many as 21 or $65.6 \%$ with 20 females or $62.5 \%$. In the nonmetastatic group, it was found that the age group of < 55 years was 8 or $72.7 \%$ with 9 females or $81.8 \%$. Based on stage $T$, it was found that the highest group was in T3 with 16 cases or $50 \%$ in the metastatic group and 6 cases in the non-metastatic group or $54.5 \%$. The sample characteristics are described in Table 1.

CXCR4 expression was stained in the membrane and cytoplasm of tumor cells (Figure 1). The results showed that CXCR4 expression with score 2 (percentage > 50\%) was mostly found in patients with regional cervical lymph node metastasis (24 cases, 75\%). Mann-Whitney test showed a significant CXCR4 expression difference in PTC with and without regional cervical lymph node metastasis ( $p=0.007)$. Regional cervical lymph node metastasis was significantly correlated with CXCR4 expression ( $p=0.006)$, with a correlation coefficient value of -0.415 .

MMP-9 expression was stained in the cytoplasm of tumor cells (Figure 2). The results showed that MMP9 expression with score 3 (percentage $>50 \%$ ) was mostly found in patients with regional cervical lymph node metastasis (21 cases, 75\%). Mann-Whitney test showed a significant MMP-9 expression difference in PTC with and without regional cervical lymph node metastasis $(p=0.030)$. Regional cervical lymph node metastasis was significantly correlated with MMP-9 expression ( $p$ $=0.027$ ), with a correlation coefficient value of -0.336 .

Spearman correlation test showed a significant correlation between CXCR4 and MMP9 expressions ( $p$ $=0.001$ ) with a correlation coefficient of 0.468 . This proved that CXCR4 expression increased along with MMP-9 expression (Figure $\mathbf{3}$ ). 


\section{Table 1.}

Characteristics

of research samples

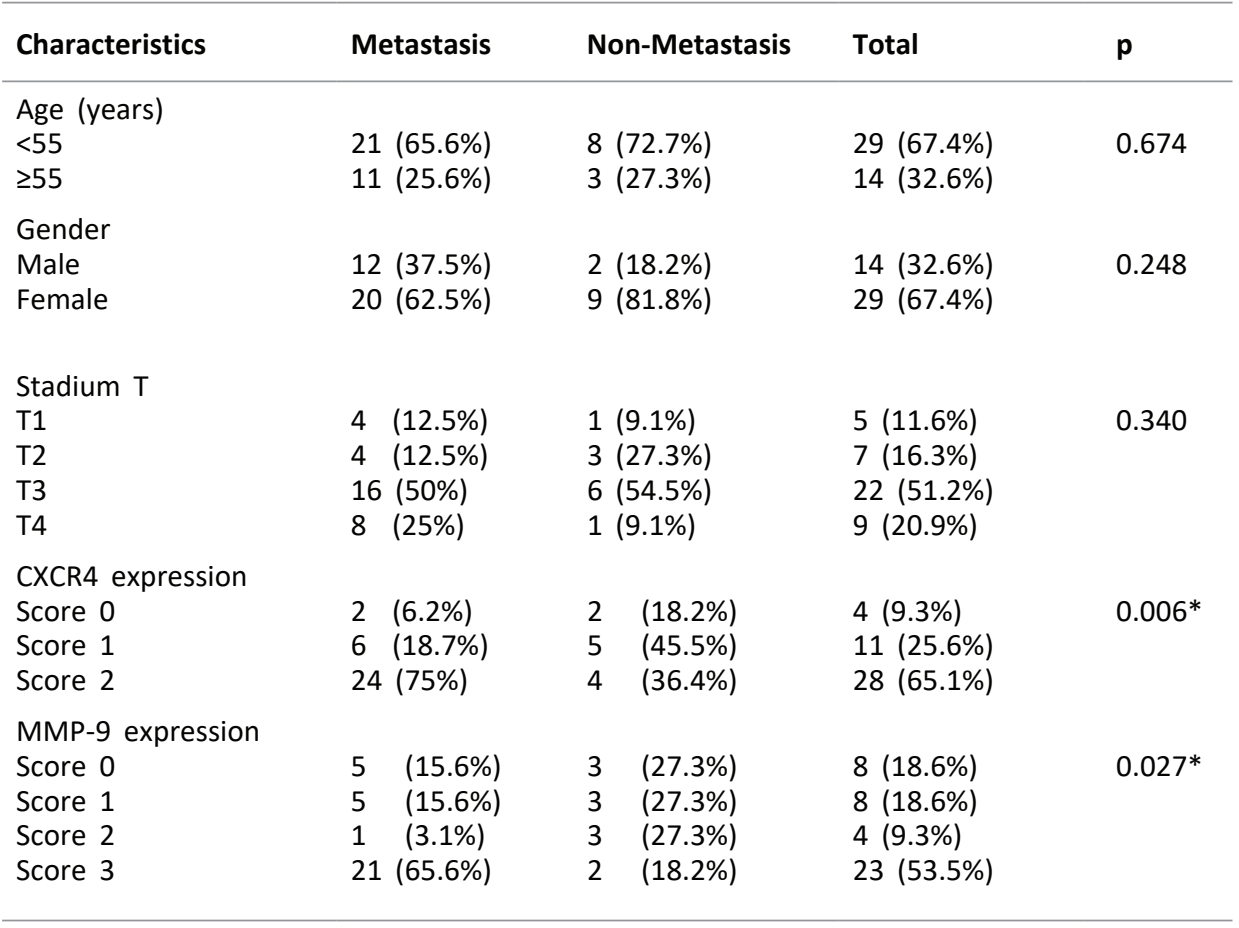

*statistically significant $(p<0.05)$
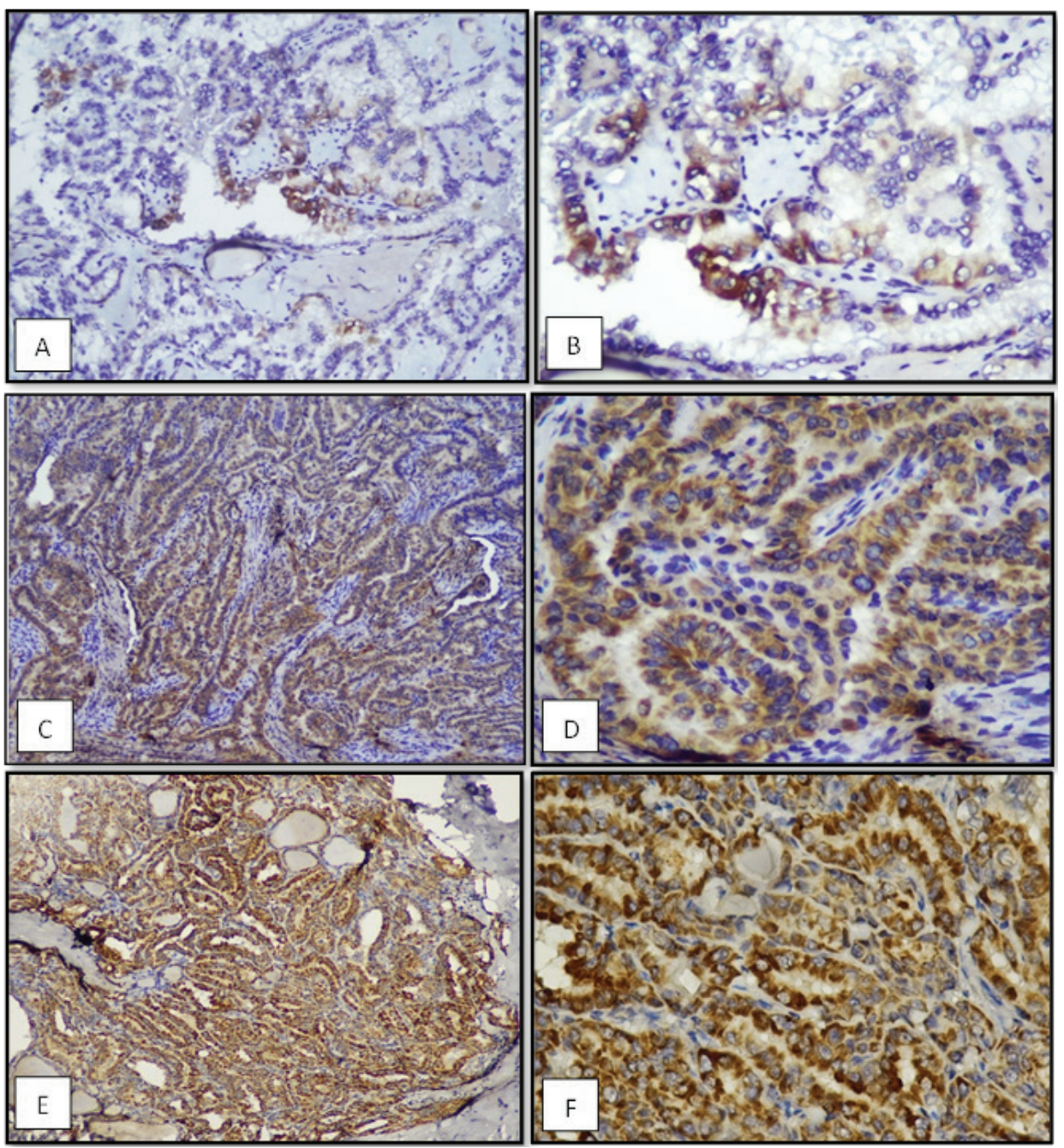

CXCR4 expression in the membrane and or cytoplasm of tumor cells. (A) and (B) CXCR4 expression score 0 , percentage $0-10 \% \%$ (magnification of 100x and 400x). (C) and (D) CXCR4 expression score 1 , percentage $11-50 \% \%$ (magnification of 100x and 400x). (E) and (F) CXCR4 expression score 2 , with percentage $>50 \%$ (magnification of 100x and 400x).

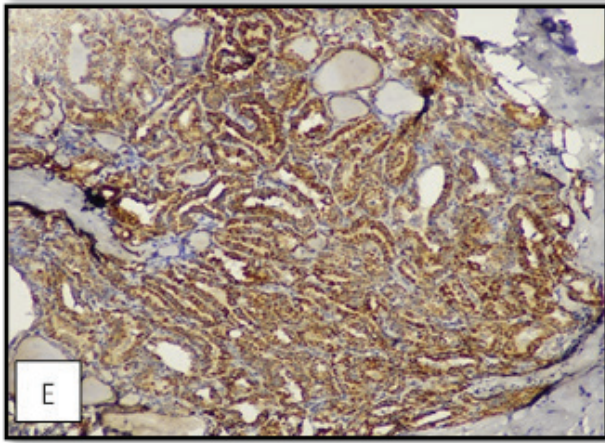


Figure 2.

MMP-9 expression in the cytoplasm of tumor cells. (A) and (B) MMP-9 expression score 0 , with percentage $<5 \%$ (magnification of 100x and 400x). (C) and (D) MMP-9 expression score 1 , percentage $5-25 \%$ (magnification of 100x and 400x). (E) and (F) MMP-9 expression score 2, percentage $26-50 \%$ (magnification of 100x and 400x). (G) and (H) MMP-9 expression score 3, percentage $>50 \%$ (magnification of 100x and 400x).
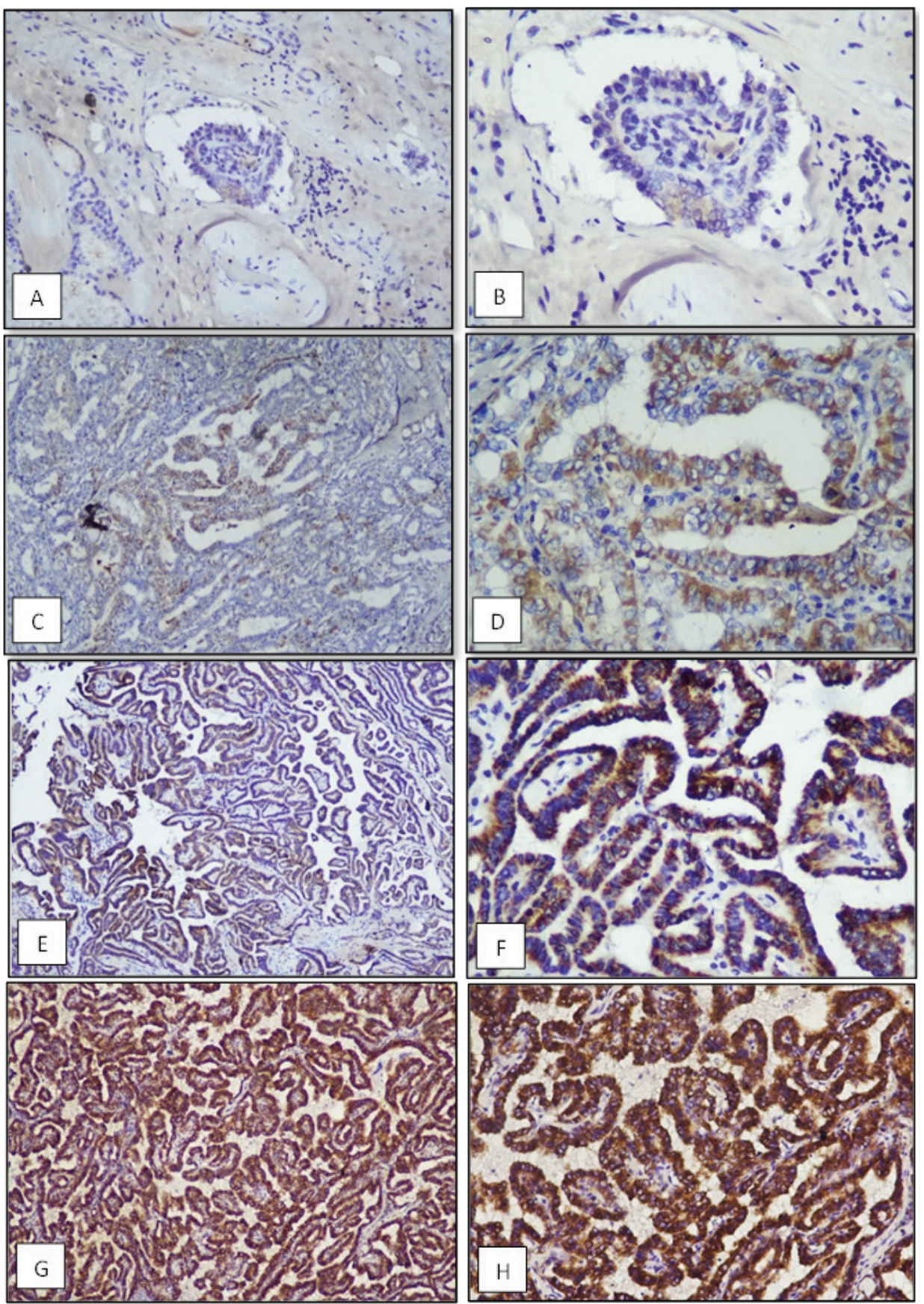

\section{DISCUSSION}

In this study, the expression of CXCR4 represented by a percentage score showed a difference with an increasing tendency in the metastatic group. The results of the Mann Whitney test showed that there were significant differences in the expressions of CXCR4 in PTC with and without metastases of neck regional lymph nodes $(p=0.007)$. The results of the Spearman correlation test showed $r$ of -0.415 with $p=0.006(p<0.05)$, meaning that there was a correlation between CXCR4 expression and the status of neck regional lymph node metastases in PTC. The higher expression of CXCR4 is in line with neck regional lymph node metastases. A negative rs value was associated with coding where the metastatic group was in the low-value group or 1 (Figure 3).

The results of this study are in line with those of several previous studies. The study by Torregrossa et al. [16] assessing the expression of CXCR4 using quantitative real-time polymerase chain reaction (Q-RT-PCR) in tumor tissue of PTC patients, showed that CXCR4 expression was higher in PTC with lymph node metastasis. The study by Lin et al. [11] found the relationship between CXCR4 expression and lymph node metastasis in patients with stage III-IV PTC $(p<0.05)$. The study by Dayer et al. [17] using Q-RT-PCR to evaluate the expression of CXCR4 


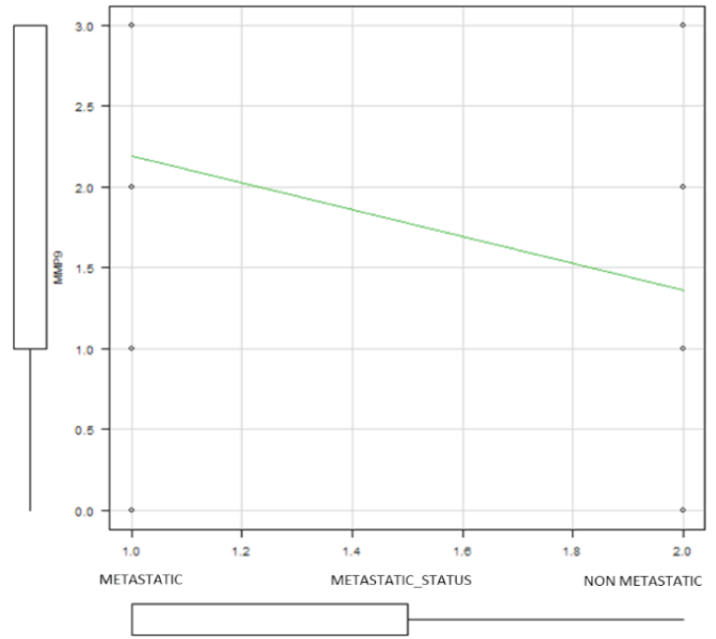

(A)

Figure 3. Scattered plot.

(A) Correlation CXCR4 expression with and without regional cervical LNM.

(B) Correlation MMP-9 expression with and without regional cervical LNM.

(C) Correlation between CXCR4 and MMP-9 expression with regional cervical LNM in PTC.

in tumor tissue of patients with invasive breast cancer, showed that CXCR4 expression was higher in breast cancer with axillary lymph node metastasis.

CXC Chemokine receptor 4 is a transmembrane protein of the G-protein-coupled cell surface receptors (GPCR) family. CXC Chemokine receptor 4 is a physiological receptor of CXC chemokine stromal cell-derived factor (SDF-1, also known as CXCL12 as the ligand) [16]. CXC Chemokine Receptor 4 is expressed in various tissues, including brain tissue, lymph node, intestine, monocyte, $B$ cell, naive T cell, and early hematopoietic progenitor cells of the immune system. Overexpression of CXCR4, caused by upregulation of Hypoxia Inducible Factor 1 Alpha (HIF-1 $\alpha$ ), is found in $>20$ types of malignancy. Tumor cells expressing CXCR4 tend to migrate to organs with increased CXCL12. Under hypoxic conditions, the von Hippel-Lindau (VHL) tumor suppressor gene, which induces the degradation of HIF-1, is inactivated; thus, elevated levels of HIF-1 stimulate CXCR4 expression via the VHL-HIF-1 pathway [17].

The binding of CXCR4 and CXCL12 activates several intracellular signaling pathways controlling cell chemotaxis, survival, proliferation, and migration of tumor cells [17].

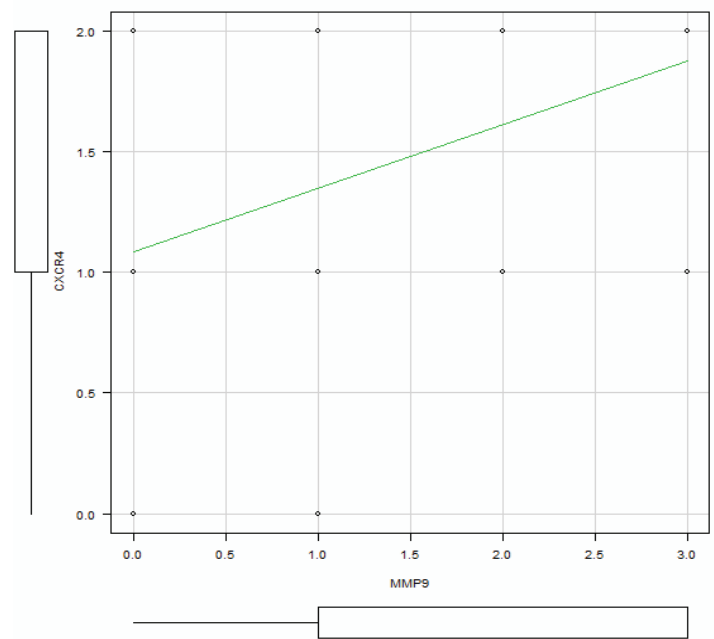

(B)

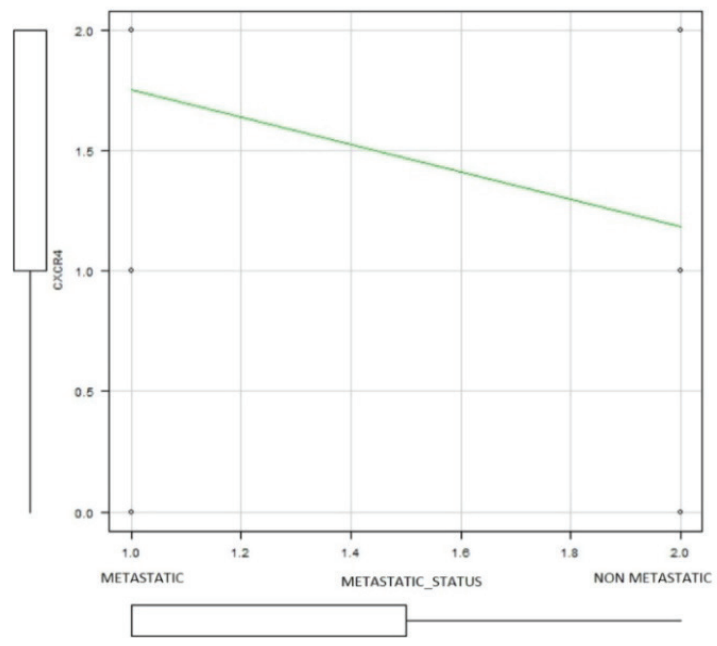

(C)

As a G-protein-coupled receptor (GPCR), the activation of CXCR4 receptor is mediated by inner plasma membrane-related intracellular heterotrimeric G-protein. $\mathrm{G}$ heterodimeric protein consists of $\mathrm{G} \alpha, \mathrm{G} \beta$, and $\mathrm{G} \gamma$ subunit. In a basal or inactive state, $\mathrm{G} \alpha$ subunit contains guanine nucleotide diphosphate (GDP). Upon activation, $\mathrm{G} \alpha$ subunit is bound to guanine nucleotide triphosphate (GTP) which will then activate PI3K. PI3K activation induces signaling cascade leading to Akt activation, which will then induce transcription factor-KB (NF-KB) $[10,16,17]$.

CXCR4 protein may increase tumor cell metastasis through the angiogenesis process. The bond between CXCR4 and CXCL12 will activate endothelial progenitor cells to migrate surrounding tumor cells and create new vessels. CXCR4 is also related to VEGF expression and platelet-derived growth factor D (PDGF-D) which correspond with the formation and stabilization of new vessels around tumor cells. The formation of new vessels around tumor cells will enhance tumor cell metastasis $[18,19,20]$. CXCR4 might also affect the immune system around tumor cells. CXCR4 protein will attract dendritic cells to migrate around tumor cells. Dendritic cells can suppress anti-tumor immunity by inactivating cytotoxic 
T-cell lymphocytes [21]. The signal from CXCR4/CXCL12 will induce epithelial to mesenchymal transition (EMT) by activating ERK1/2 pathway, enhancing MMP activation to degrade ECM around tumor cells. The basal membrane of endothelial venules is also rich in CXCL12; therefore, they will invite malignant cells which express CXCR4 to enter blood circulation resulting in distant metastasis [22]. The study by Lin et al. [11] made a comparison between the two-year survival rates of patients with osteosarcoma expressing CXCR4 and those not expressing CXCR4. They found that the two-year survival rate of CXCR4- positive patients $(32.4 \%)$ was significantly lower than that of CXCR4-negative ones (78.9\%). Given the negative effects of CXCR4 expression on survival, it has been suggested that CXCR4 may be used as a potential prognostic factor [13].

In this study, the expression of MMP-9 represented by a percentage score showed a difference with an increasing tendency in the metastatic group. The results of the Mann Whitney test showed that there were significant differences in the expressions of MMP-9 in PTC with and without metastases of neck regional lymph nodes $(p=0.030)$. The results of the Spearman correlation test showed $r s=-0.336$ with $p=0.027(p<0.05)$, meaning that there was a correlation between MMP-9 expression and the status of neck regional lymph node metastases in PTC. The higher expression of MMP-9 is in line with neck regional lymph node metastases. A negative rs value was associated with coding where the metastatic group was in the low-value group or 1 (Figure 3).

The result of this study was consistent with a study by Liu et al. [23] which also found a significant correlation between MMP-9 expression and central and lateral lymph node metastasis in the late-stage PTC (stage IIIIV) ( $<<0.05)$. A study by Hwang et al. [24] investigating MMP-9 in gastric cancer, also reported a similar result where MMP-9 was correlated with lymph node metastasis and lymphatic invasion, and bad prognosis. Liabakk et al. [25] found that MMP-2 and MMP-9 levels increased significantly with the cancer stage. Parson et al. [26] observing gelatinase enzyme (MMP-2, MMP-9), showed that overexpression in gastrointestinal malignancy was related to its role in tumor invasion and metastasis $[20,26]$

Matrix metalloproteinases-9 (MMP-9) is broadly divided according to substrate specificity, divided into 6 groups namely collagenases (MMP-1, 8, 13), gelatinases (MMP-2 and MMP-9), stromelysins (MMP-3, 10, 11), Matrylisins (MMP-7 and MMP-26), membrane-type MMP (MMP-14, 15, 16, 17, 24 and 25), and other MMPs. MMP-9 is known as 92 kDa gelatinase. MMP-9 secreted by tumor cells is related to tumor metastasis; therefore, MMP-9 can degrade collagen types IV, V, VII, and X. Type IV collagen is the main collagen supporting basal membrane and is especially abundant in vascular endothelium in the basement membrane, and it acts as a barrier to invasion and metastasis. The high expression of MMP-9 causes degradation of the extracellular matrix making it easier for tumor cells to invade and metastasize $[14,27,28]$. Increased expression of MMP-9 can be a useful diagnostic marker and maybe a potential target in the treatment of thyroid carcinoma. Previous studies have shown MMP-9 to have good sensitivity and specificity for the diagnosis of PTC [23].

The correlation between CXCR4 and MMP-9 expressions in PTC with and without neck regional LNM was statistically tested using the Spearman correlation test; the result obtained was $r s=0.468$ with $p=0.001$ $(p<0.05)$. This proves that there is a significantly positive correlation between the expressions of the two; the higher the expression of CXCR4 is, the higher the expression of MMP-9 will be (Figure 3 ).

A study by Liang et al. [29] also found overexpression of CXCR4 and MMP-9 in 23 out of 27 cases of PTC with lymph node metastasis, with a statistically significant difference between PTC with and without lymph node metastasis $(p<0.05)$. Hao et al. [30] proved that there was a significant correlation between CXCR4 and MMP9 and lymph node metastasis in breast carcinoma with a correlation coefficient of 0.479 and $p<0.001$ ( $p<$ 0.05). A study by Zuo et al. [31] showed that CXCR4 overexpression in lung carcinoma increased EGFR expression and MMP-9 production, which then caused migration, invasion, and metastasis of tumor cells.

CXCR4 activation will trigger various signaling pathways underneath. The activated CXCR4 will split into $G \alpha$ and $G \beta \gamma$ sub-units. The $\beta \gamma$ subunit induces phospholipase-c (PLC) which will make phosphatidylinositol 4,5-biphosphate (PIP2) produce second messengers, namely diacylglycerol (DAG) and inositol triphosphate (IP3). IP3 will increase intracellular calcium excretion from the endoplasmic reticulum, which together with DAG will activate protein kinase $C$ (PKC) and MAPK/ ERK1/2 [10]. The MAPK/ERK $1 / 2$ pathway will activate the IKB kinase (IKK) enzyme complex which will phosphorylate the inhibitor IKB protein. Phosphorylation will make ІкB dissociate from NF-к $\beta$. "Free" NF-к $\beta$ will migrate into the cell nucleus to play a role in the protein translation process. The activity of NF-K $\beta$ induces the expression of MMP-9 leading to cell proliferation, angiogenesis, and metastasis of tumor cells [23,32].

Another pathway that can play a role in tumor cell migration is PI3K. PI3K can be activated by both $\mathrm{G} \beta \gamma$ and $G \alpha$ subunits and can activate the Akt and NF-k $\beta$ pathways. The PI3K/AKT pathway plays a role in angiogenesis and tumor cell proliferation, while the NF$\mathrm{\kappa} \beta$ pathway suppresses apoptotic signals by upregulating the $\mathrm{Bcl}-2$ gene. Chinni et al. [33] reported that CXCL12 induces the expression of MMP-9 which is regulated by NF-KB activity in prostate cancer cells by activating the PI3K-Akt-NF-KB and MEK pathways $[13,33]$. In this study, similar results were obtained showing that the 
overexpression of CXCR4 can increase MMP-9 production, which ultimately results in increased cell migration and invasion.

The limitation of this study is that only two biomarkers were analyzed, whereas for all malignancies, in this case of PTC, many other pathways and biomarkers play a role in complex interactions that will ultimately affect tumor proliferation, invasion, and metastatic processes. In addition, the limitations of this study are the very small number of samples in the group without metastases and the deviation value in this study, which is quite large, namely $15 \%$.

\section{CONCLUSIONS}

CXCR4 and MMP-9 expressions were proven to correlate with regional cervical lymph node metastasis in papillary thyroid carcinoma. Both can be used as potential prognostic factors, either concurrently or separately.

\section{DECLARATIONS}

\section{Competing of Interest}

The authors declare no competing interest in this study.

\section{Ethical Approval}

This study had been approved by the Ethical Committee of Dr. Soetomo Hospital Surabaya (0050/LOE/301.4.2/ VI/2020).

\section{Acknowledgment}

The authors wish to thank Dr. Budi Utomo, Department of Public Health and Preventive Medicine, Universitas Airlangga for statistical analysis.

\section{REFERENCES}

1. Bu R, Siraj AK, Divya SP, et al. Telomerase reverse transcriptase mutations are independent predictor of disease-free survival in middle eastern papillary thyroid cancer. Int J Cancer. 2018;142(10):2028-39.

2. Bray F, Ferlay J, Soerjomataram I, et al. Global cancer statistics 2018: GLOBOCAN estimates of incidence and mortality worldwide for 36 cancers in 185 countries. Ca Cancer J Clin. 2018;68(6):394-424.

3. Pusat Data dan Informasi Kementerian Kesehatan RI. Infodatin. Jakarta. 2015. https://pusdatin.kemkes. go.id.

4. Paulino TL, Holt E, Callender GG, et al. Management of Papillary Thyroid Cancer: An Overview for the Primary Care Physician. J Clin Outcomes Manag. 2014;21(5).

5. Jin $W X$, Jin $Y X, Y e D R$, et al. Predictive factors of skip metastasis in papillary thyroid cancer. Med Sci Monit. 2018;3(24):2744-9.
6. Tallon P, Holt E, Callender G, et al. Management of papillary thyroid cancer: an overview for the primary care physician. J Clin Outcomes Manag. 2014;21(5):233-40.

7. Sun $W$, Lan $X$, Zhang $H$, et al. Risk factors for central lymph node metastasis in CNO papillary thyroid carcinoma: a systematic review and meta-analysis. PloS ONE. 2015;10(10):e0139021.

8. Orbach GO. Risk stratification in differentiated thyroid cancer: An ongoing process. Rambam Maimonides Med J. 2016;7(1):e0003.

9. Gonza' lez HE, Leiva A, Tobar H, et al. Altered chemokine receptor expression in papillary thyroid cancer. Thyroid. 2009;19(9):957-65.

10. Teicher BA, Fricker SP. CXCL12 (SDF-1)/CXCR4 pathway in cancer. Clin Cancer Res. 2010;16(11):2927-31.

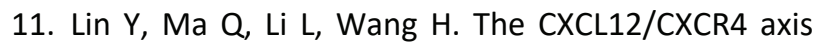
promotes migration, invasion and EMT in human papillary thyroid carcinoma B-CPAP cells via NF-KB signaling. Biochem Cell Biol. 2018;96(5):619-26.

12. Hua $\mathrm{H}$, Li M, Luo $\mathrm{T}$, et al. Matrix metalloproteinases in tumorigenesis: an evolving paradigm. Cell Mol Life Sci. 2011;68(23):3853-68.

13. Liao $\mathrm{YX}$, Zhou $\mathrm{CH}$, Zeng $\mathrm{H}$, et al. The role of the CXCL12-CXCR4/CXCR7 axis in the progression and metastasis of bone sarcomas (Review). Int J Mol Med. 2013;32(6):1239-46.

14. Luo D, Chen H, Li X, et al. Activation of the ROCK1/ MMP-9 pathway is associated with the invasion and poor prognosis in papillary thyroid carcinoma. Int J Oncol. 2017;51(4):1209-18.

15. Meng $X$, Hua $T$, Zhang $Q$, et al. Expression and clinical significance of matrix metalloproteinase 9 (MMP9) papillary thyroid carcinomas. Afr J Pharm Pharmacol. 2012;6(44):3075-9.

16. Torregrossa L, Giannini R, Borrelli N, et al. CXCR4 expression correlates with the degree of tumor infiltration and BRAF status in papillary thyroid carcinomas. Mod Pathol. 2011;25(1):46-55.

17. Dayer R, Babashah S, Jamshidi S, Sadeghizadeh M. Upregulation of CXC chemokine receptor 4-CXC chemokine ligand 12 axis in invasive breast carcinoma: A potent biomarker predicting lymph node metastasis. J Can Res Ther. 2018;4(2):345-50.

18. Cojoc M, Peitzsch C, Trautmann F, et al. Emerging targets in cancer management: role of the CXCL12/ CXCR4 axis. Onco Targets Ther. 2013;6:1347-61.

19. Hiller D, Chu QD. CXCR4 and axillary lymph nodes: review of potential biomarker for breast cancer metastasis. Int J Breast Cancer. 2011;(2011):1-6.

20. Domanska UM, Kruizinga RC, Nagengast WB, et al. A review on CXCR4/CXCL12 axis in oncology: No place to hide. Eur J Cancer. 2013;49(1):219-30.

21. Xu C, Zhao H, Chen $H$, and Yao Q. CXCR4 in breast cancer: oncogenic role and therapeutic targeting. Drug Des Devel Ther. 2015;9:4953-64. 
22. Guo F, Wang Y, Liu J, et al. CXCL12/CXCR4 a symbiotic bridge linking cancer cells and their stromal neighbors in oncogenic communication networks. Oncogene. 2015;35(7):816-26.

23. Liu X, Su C, Xu J, et al. Immunohistochemical analysis of matrix metalloproteinase- 9 predicts papillary thyroid carcinoma prognosis. Oncol Lett. 2018;17(2):2308-2316.

24. Hwang TL, Lee LY, Wang CC, et al. Claudin-4 expression is associated with tumor invasion, MMP2 and MMP-9 expression in gastric cancer. Exp Ther Med. 2010;1(5):789-97.

25. Liabakk NB, Talbot I, Smith RA, et al. Matrix metalloprotease 2 (MMP-2) and matrix metalloprotease 9 (MMP-9) type IV collagenases in colorectal cancer. Cancer Res. 1996;56(1):190-6.

26. Parsons SL, Watson SA, Collins HM, et al. Gelatinase (MMP-2 and MMP-9) expression in gastrointestinal malignancy. Br J Cancer. 1998;78(11):1495-502.

27. Vandooren J, Van den Steen PE, Opdenakker G. Biochemistry and molecular biology of gelatinase $B$ or matrix metalloproteinase-9 (MMP-9): the next decade. Crit Rev Biochem Mol Biol. 2013;48(3):222-72.

28. Conlon GA, Murray GI. Recent advances in understanding the roles of matrix metalloproteinases in tumour invasion and metastasis. J Pathol. 2019;247(5):629-40.
29. Liang H, Zhong $\mathrm{Y}$, Luo $\mathrm{Z}$, et al. Assessment of biomarkers for clinical diagnosis of papillary thyroid carcinoma with distant metastasis. The International Journal ff Biological Markers. 2010;25(1):38-45.

30. Hao L, Zhang C, Qiu Y, et al. Recombination of CXCR4, VEGF, and MMP-9 predicting lymph node metastasis in human breast cancer. Cancer Lett. 2007;253(1):34-42.

31. Zuo J, Wen M, Li S, et al. Overexpression of CXCR4 promotes invasion and migration of non small cell lung cancer via EGFR and MMP 9. Oncol Lett. 2017;14(6):7513-21.

32. Sticker TP and Kumar V. Neoplasia. In: Kumar V, Abbas AK, Fausto N, Aster JC, editors. Robbins pathology basic of disease. 8th ed. Philadelphia: Saunders; 2010. p259-330.

33. Chinni SR, Sivalogan S, Dong Z, et al. CXCL12/CXCR4 signaling activates Akt-1 and MMP-9 expression in prostate cancer cells: The role of bone microenvironment-associated CXCL12. Prostate. 2006;66(1):32-48. 